\title{
Beiträge zur Pathologie der Psoriasis.
}

\author{
Von \\ Dr. Josef Schütz \\ in Frankfurt a. Main. \\ (Hierzu Tafel XIII.)
}

In den letzten Jahren habe ich mir über 100 Fälle von Psoriasis, welche sämmtlich meiner Privatpraxis entstammen und einer eingehenden Beobachtung unterlagen, Aufzeichnungen gemacht. Die Ergebnisse weichen vielfach ron dem ab, was andere und ich an poliklinischem Material früher fanden. Zum Theil bestätigen sie in erhöhtem Masse einzelne schon verbreitete Anschauungen. Die Dunkelheit, welche bis zur Stunde über die Aetiologie der so hartnäckigen Krankheit herrscht, rechtfertigt es, gewonnene Anhaltspunkte und Beobachtungen immer wieder zu sammeln und der allgemeinen Beurtheilung zugänglich zu machen. Von den Bausteinen, welche einzeln und mühsam von vielen tüchtigen Beobachtern in langer Zeit zusammengetragen wurden, um ein einheitliches plastisches Gebilde von der Wesenheit der Psoriasiserkrankung zu gewinnen, liegen noch viele unbenutzt, weil sie sich nicht einfügen liessen nach der jeweilig herrschenden Anschauung über die Erkrankung, oder weil passende Zwischenstücke nocb fehlten. Mögen meine kleinen Beiträge die eine oder andere Lï̈cke ausfüllen und das Ganze festigen helfen. 
Weitverbreitet ist die Ansicht, dass Psoriasis gleichhäufig beim männlichen und weiblichen Geschlecht angetroffen werde. Die meisten Lehrbücher und Autoren sprechen sich über diesen Punkt nicht aus oder verfallen sofort einer oppositionellen Kritik. F. Hebra erwähnt in seinem Lehrbuch nur ganz beiläufig, dass er jährlich unter circa 3000 Hautkranken 50 Psoriasiskranke (33 M. +17 W.) sehe, ohne diesem sonderbaren Verhältniss irgendwelchen Werth beizumessen, was mit Ruicksicht auf poliklinisches Material vielleicht zweckmässig erscheinen kann, da Frauen überhaupt erfahrungsmässig zur Poliklinik ein geringeres Contingent stellen als Männer. Unter meinem Material aus der Privatpraxis kamen auf 66 Patienten männlichen Geschlechts 34 Frauen und Mädchen, also doppelt soviele männlichen Geschlechts als Weibliche, eine Zahl, welche mit derjenigen, welche F. Hebra beiläufig erwähnt, genau übereinstimmt. Wir werden später hierauf zurückkommen und wollen diese Thatsache einstweilen als solche vormerken.

Dem Alter nach standen 95 meiner Patienten zur Zeit des zumeist anamnestisch eruirten ersten Ausbruchs der Krankheit zwischen dem 9. und 24. Lebensjahr. Es bestätigt dies die alte Erfahrung, dass Psoriasis in den Entwicklungsjahren mithin zur Zeit des Wachsthums des Skelets und der Musculatur, der Entwicklung der Spannungsverhältnisse in der Haut, auftritt. Einmal sah ich Psoriasis bei einem Herrn erst im 64. Lebensjahr auftreten. Denselben kenne ich persönlich seit langen Jahren. Da derselbe ängstlicher Natur ist und sorgsam auf seine Gesundheit und insbesondere auf sein Aeusseres achtet, so kann man kaum annehmen, dass frühere Psoriasisausbrüche unbemerkt geblieben sein sollten. Es standen einzelne numuli am Halse und der Brust. Eine Verwechselung mit Eczema seborrhoicum war nicht möglich. Der behaarte Kopf war frei. Sodann sah ich Psoriasis an beiden Knien bei einem 13 Monate alten Mädchen auftreten, während ich deren Schwester von nur 6 Jahren an einer etwa $1 \mathrm{Jahr}$ lang bestehenden Psoriasis des Stammes behandelte. Endlich behandelte ich zwei Brüder (von 11 und 10 Jahren), deren universelle Psoriasis nach Aussage der Mutter seit dem zweiten Lebensjahre bestehen sollte. 
Heredität ïberhaupt konnte ich in 28 Fällen nachweisen. Zwölfmal waren bei Psoriasispatienten die Eltern ebenfalls an Psoriasis erkrankt, und zwar waren in nur 4 Fällen von den Eltern der mit den betreffenden psoriasiskranken Nachkommen an Geschlecht nicht übereinstimmende Theil erkrankt. Achtmal habe ich Psoriasis unter Geschwistern beobachtet, fünfmal bei Schwestern bez. Geschwisterkindern. In einem Falle waren erkrankt ausser dem männlichen Psoriasiskranken selbst dessen Mutter und deren Brüder, in einem anderen Falle dessel. Tante. Ein Ueberspringen der Psoriasis von den Grosseltern auf die Enkel beobachtete ich selbst nie.

Zusammengefasst beobachtete ich also Auftreten von Psoriasis

I. in horizontaler Linie der Stammbäume

a) bei Geschwistern,

b) bei Geschwisterkindern,

II. in absteigender Linie ersten Grades

a) bei Eltern und Kindern,

b) bei Onkeln und Tanten einerseits und Neffen bez. Nichten andererseits.

Eine Bevorzugung derselben Geschlechtslinie bei der Heredität konnte nicht nachgewiesen werden (unter Berücksichtigung des selteneren. Vorkommens der Psoriasis beim weiblicben Geschlecht überhaupt).

Wie schwer es ist, die Heredität manchmal festzustellen, mag aus Folgendem erhellen: Es trat in meine Behandlung eine junge Dame mit einer Erkrankung der galea, welche ich als Eczema seborrhoicum auffassen zu müssen glaubte. Die eingeleitete Behandlung mit Resorcinkaliseife und Schwefelsalicylsalbe fruchtete nicht. Während der Behandlung zeigt mir die Mutter der Dame eine Erkrankung ihres rechten Daumennagels, welche mir sofort psoriatischen Ursprungs zu sein schien. In Folge dessen erkundigte ich mich weiter nach Hauterkrankungen in der Familie und hörte nun zur Lösung des Räthsels, dass der zu einer Dienstleistung beim Militär abwesende Sohn an tiichtiger Psoriasis des Stammes leide. Die nun eingeleitete Arsen- und Chrysarobinbehandlung brachte Schwund der Symptome. Den Sohn behandelte ich später 
gleichfalls mit Erfolg. Aus derartigen Recherchen, welche Polikliniken mehr oder weniger entgehen müssen, wird mein relativ hoher Procentgehalt heredit. Psoriasis gegenïber gegentheiligen Behauptungen mancher Autoren eine leichte Erklärung finden. Auch die Allgemeinconstitution meiner Clientel zeigte namentlich bezüglich des Hautorgans übereinstimmende Momente.

In allen Fällen ohne Ausnahme bestand eine relativ starke Behaarung sowohl des Kopfes wie des Körpers, die namentlich an den Streckseiten der Arme und an der Schulterblattgegend oft besonders auffiel. Zumal bei weiblichen Individuen, welche an Psoriasis erkrankten, war die sichtbare Lanugo stets gut ausgeprägt. Bei zweien (Alter 21 und 26 Jahre) bestand sogar ein über jeden höflichen Zweifel erhabener Schnurr- und Backenbart. Bei Männern waren Haupt- und Barthaar entweder gut entwickelt und dicht oder doch früher stark gewesen. Bei 3 Patienten mit etwas gelichtetem Haupt (wahrscheinlich in Folge unsinnigen Gebrauchs stark alkoholischer Tinkturen und sonstiger Friseurkünste) zeigte eine überaus starkeBartentwicklung die von Haus aus reiche Veranlagung des Haarbodens, eine Wechselbeziehung, welche überhaupt gerne eintritt und schon Eble bekannt war.

Der Farbe nach war das Haar:

$\begin{array}{lrrc}\text { schwarz } & \text { bei } & 18 & \text { Personen } \\ \text { dunkelbraun } & " & 57 & " \\ \text { hellblond } & " & 25 & " \\ \text { roth } & " & 0 & "\end{array}$

Das Fehlen der Psoriasis bei den allerdings immer seltener werdenden Rothhaarigen ist hier statistisch auffallend und erinnert unwillkürlich an die bekannte Zartheit der Haut derselben. Der oft geübte Spott über rothes Haar erweist sich hier wieder einmal als unangebracht.

Complicirende Hauterkrankungen bei den Psoriasispatienten waren sechsmal Acne (4mal des Rückens), viermal Lichen pilaris der Extremitätenaussenseite, fünfmal Furunkel des Nackens (sog. Stehkragenfurunkulose). Complicirendes Eczem (nicht arteficiellen bezügl. arzneilichen Ursprungs) sah ich nie, erinnere mich auch nicht, früher je bei Leuten mit Psoriasis gesehen zu haben. 
Durchweg waren die Psoriasiskranken im allgemeinen gesunde, schöne Personen, wie F. Hebra treffend schreibt "von straffer Muskelfaser." Bei den weiblichen Individuen konnte man, abgesehen von der bereits erwähnten stärkeren Behaarung, auch bezügl. des Skeletts und der Musculatur eine Annäherung an den männlichen Typus manchmal constatiren.

Nur einer meiner Patienten zählte zu den fettleibigen, Marienbad alljährlich heimsuchenden Phlegmatikern. Hier zeigten die Psoriasisefllorescenzen eine sehr spärliche Röthe, dieselben waren fast von citronengelber Farbe, die Schuppung gering. Bäder, Theerseife und Theerpinselungen genügten, um in wenigen Wochen die Krankheit zum Verschwinden zu bringen, während bei seinem psoriasiskranken mageren Sohn eine recht hartnäckige Ausbreitung und ein langwieriger Verlauf sich geltend machten.

Einmal war Psoriasis mit Diabetes complicirt, der langwierigste Fall der ganzen Reihe. Die Behandlung musste wegen der Unverträglichkeit der meisten Mittel mit Arsen und Pyrogallusspiritus allein durchgeführt werden und dauerte drei Jahre. Zweimal sah ich Psoriasis im Verein mit Tuberculose, nach Hebra ein äusserst seltenes Ereigniss: einen Mann mit Kehlkopftuberculose, eine Frau mit Tuberculose der platten Schädelknochen. Letztere wurde mit Giück hier in Frankfurt ausgemeisselt; gegenwärtig sieht die Patientin aber nach brieflicher Mittheilung ihrem Ende durch tuberculöse Peritonitis und detto-Leberabscess entgegen. Auffallenderweise war die mit Tuberculose gepaarte Psoriasis in beiden Fällen durchaus nicht unvollkommen ausgebildet, sondern mit starker Röthe und Schuppung einhergehend.

Die erste Entstehung der Psoriasis wird von den Patienten und deren Angehörigen manchmal auf acute Erkrankungen und Arzneieinwirkungen zurückgefïhrt. Ueberstandene Masern, sehr oft Keuchhusten, Bronchialcatarrhe, reizende Salzbadecuren spielen hier die Hauptrolle und werden im Sinne der Humoralpathologie, die nun einmal aus den Ideen des Volkes. nicht schwinden will, verwerthet. Die Angaben scheinen mir nicht in jedem Falle einwurfsfrei, deshalb will ich an anderer 
geeigneter Stelle später nur wenige eigene Beobachtungen einflechten.

Besondere Aufmerksamkeit widmete ich der Localisation. Obwohl man in der Privatpraxis erklärlicherweise hier auf mancherlei Schwierigkeiten stösst, war ich doch so glücklich, an die 50 Fälle im Schema einzutragen und auch eine Anzahl Recidive in ihrer Ausbreitung graphisch zu fixiren. Die Ergebnisse sind kurz folgende:

1. Psoriasis kennt im allgemeinen keine Körperstellen, an denen sie gelegentlich nicht aufträte, namentlich ein ausschliessliches Befallensein der Streckseiten ist nicht wahrzunehmen. 2. Recidive kehren sich im allgemeinen nicht streng an die Localisation früherer Ausbriche; wo aber dieselben Stellen wiederholt betroffen werden, ist die Psoriasis von grosser Hartnäckigkeit. 3. Die Ausbreitung ist meist symmetrisch.

Bei Durchsicht der Schemata, in welchen die Localisation der Psoriasis verzeichnet wurde, fand ich den bei der einfachen Beobachtung gewonnenen Eindruck bestätigt, dass die Psorias sich gerne an Stellen findet, an welchen 4 . die Haut besonders straff angeheftet ist und einen naheliegenden Knochen zur Unterlage hat (Brustbein, Kreuzbein, Schienbein, galea, Ohr, Stirn, Hand und Fussrücken u. s. w. und 5. an Stellen, deren Flächenwachsthum gegen ihre Umgebung beim Wachsen des Körpers zurückbleiben (fixe Punkte der Articulationen und deren Umgebung: Knie, Ellenbogen, Fingerknöchel). Es sind dies fixe, oft symmetrische Oertlichkeiten, welche beim Menschen und mehr noch analog beim Thier durch starke Behaarung und eigne Haarwirbelbildung ausgezeichnet sind. Nachträglich kam mir die neue trbeit ron M. Schein ${ }^{1}$ ) zu Gesicht, in welchen diese Haarbildung mit den Spannungsverhältnissen jener Hautstellen genetisch in Zusammenhang gebracht ist, und ich muss sagen, dass das Studium jener Arbeit für jeden, welcher sich mit der Localisation der Psoriasis eingehender befassen will, förderlich sein wird. Genannte Stellen entsprechen auch vielfach den Dreiecken in den Tafeln von C. Langer (die Spaltbildungen der Haut darstellend) and den Haarwirbeln

i) M. Schein. Ueber das Wachsthum der Haut und der Haare des Menschen. Arch. f. Derm. u. Syph. 1892. p. 429. 
und Richtungsconvergenzen auf den Tafeln von C. Aug. Voigt. 6. Nur zum kleinsten Theil waren Stellen erkrankt, an denen analog den Experimenten $K$ öb n e r's und W urtzd offer's nachweislich eine mechanische oder chemische äussere Schädlichkeit (Kleiderdruck, Verbandeczem) bestanden hatte.

Fusssohlen und Handteller, obere Augenlider, Schleimhäute waren stets frei von Psoriasis. Der behaarte Kopf war 36 mal (1) Sitz der Psoriasis und viermal der zuerst befallene Körpertheil. Dieser hohe Procentsatz findet wohl auch seine Erklärung in der sorgfältigen Beobachtung, welche in der Privatpraxis Arzt und Patient ausuiben können. Die Differentialdiagnose bei solitärer Psoriasis capillitii erwies sich namentlich gegenuiber Eczema seborrhoicum und Herpes tonsurans als nicht leicht. Hier ist eine sorgfältige Anamnese und der innerliche Gebrauch grosser Dosen Arsen ohne äusserliche Therapie oft das am raschsten Aufklärende. Der negative Ausfall der mikroskopischen Untersuchung auf Pilze beweist nur, dass man nichts gefunden hat, nicht, dass keine Pilze vorhanden sind. Es ist und bleibt ein Wahrscheinlichkeitsbeweis, den man im Hinblick auf ein ev. positives Ergebniss anstellt.

Hier muss ich noch einer besonderen Form der Psoriasis gedenken, welche ich zweimal an der Stirn und Stirnhaargrenze beobachtete. Dort standen winzige Efflorescenzen äusserst dicht zusammen, so dass ein breites, rothes Band, wie es dem Druck eines Hutrandes entsprechen kann, dort zu sehen war. Die Hautschüppchen hatten ein eigenthümlich glasiges, durchsichtiges Ansehen, so dass man auf den ersten Blick durchaus keine Psoriasis, sondern ein Eczema acutum vesiculosum ror sich zu haben glaubte. Diese Form erwies sich als schwer zu heilen. Die Beseitigung erfolgte am besten durch Pyrogallussalben. Vergeblich habe ich mich in der Literatur nach einer Schilderung dieser Psoriasisform umgesehen.

Selten waren Gesicht (Augenbrauen, Nase, Nasenwangenfalten, Backen: 4 Fälle) und Handruicken (3 Fälle) erkrankt. Nur einmal war das Dorsum der letzte n Phalanx der Finger, zwischen Nagelfalz und nächster Hautquerfalte, Sitz einer Psoriasisefflorescenz.

Häufiger dagegen fand ich Psoriasis ungium. 
Schon früher, vor den jetzigen Untersuchungen, habe ich manchmal Psoriasis der Fingernägel beobachtet und mich gewundert, in der Literatur hiervon keine Beschreibung haben auffinden zu können, welche dem primären Sitz der Psoriasis ungium besondere Aufmerksamkeit widmete.

Unter den 100 auf diese Nagelerkrankung hin untersuchten Fällen von Psoriasis war ich 11 Mal in der Lage, Psoriasis ungium anzutreffen, also relativ häufig. Eine in Folge dessen unternommene Durchsicht der neueren Schriften, über Psoriasis sowohl wie über Nagelerkrankungen überhaupt, lieferte auch diesmal ein vollständig negatives Resultat. Unsere gangbaren Lehrbücher über Hautkrankheiten erwähnen die zu beschreibende primäre Erkrankung mit keinem Worte, sondern nur jene Nagelverdickungen, Verfärbung, Längs- und Querstreifen und ebensolche Wülste und Risse, wie sie überhaupt nach langwierigen schweren Hauterkrankungen (Eczem, Lichenruber, Dermatomykosen) an den Nägeln schliesslich als s e cu ndäre Veränderungen anzutreffen sind, ohne für eine dèr zu Grunde liegenden Hautkrankheiten als solche ein Charakteristikum zu bieten.

F. Hebra (Lehrb. der Hautkrankheiten, I. Bd., 1872, pag. 347), welcher der Nagelerkrankung bei Psoriasis noch am ausfïhrlichsten gedenkt, sagt: "Die Nägel werden dicker, glanzlos, uneben, dunkler gefärbt, gelb bis braun, brüchig, so dass sie nicht frei bis über die Fingerspitzen hervorragen, sondern an der Spitze abbrechen und zerklüften. In manchen Fällen merkt man im Beginne der Nagelerkrankung, wenn der Nagel selbst noch durchscheinend ist, unter demselben, also am Nagelbette, eine ähnliche punktförmige Psoriasisstelle wie an anderen Hautpartien, woraus hervorgeht, dass derselbe Process, der die Psoriasisefflorescenzen an den übrigen Stellen veranlasst, auch hier statthat."

Weyl (Ziemssen, Handb. der Hautkrankheiten, I. Bd., 1883, pag. 496) berichtet: „Die Nägel der Finger und Zehen werden bei inveterirter sehr ausgedehnter Psoriasis meist secundär mit in den pathologischen Process gezogen, eine Erscheinung, die auch bei anderen chronischen mehr universellen Dermatosen zu beobarhten ist; sie werden am freien 
Rande stark verdickt, blättern auseinander, bekommen tiefe Tüpfel, ähnlich einem Fingerhute, oder trüb gelblich weisse Flecken und Streifen; stellenwe1se splittern unregelmässig verschieden grosse Stücke, so dass zuweilen nur noch kleine entstellte Reste an der Matrix übrig bleiben. In einzelnen Fällen können die Nägel lange Zeit den ausschliesslichen und ersten Sitz des psoriatischen Processes bilden."

Geber hat in seinem Capitel „Krankhafte Veränderungen. des Nagels und des Nagelbettes" (Ziemssen, Handb. der Hautkrankheiten, II. Bd., 1884, pag. 237 u. f.) ebenfalls nur solche secundäre Veränderungen des Nagel bei Psoriasis berücksichtigt.

Auch Kaposi (Pathologie und Therapie der Hautkrankheiten, 1883, pag. 391) sieht in der Nagelerkrankung bei Psoriasis eine secundäre Affection und gibt kurz an: "Wie die Haare durch rasches Ausfallen ihre Mitleidenschaft bekunden, so erkranken auch die Nägel bei jeder lang dauernden Psoriasis in der Art, dass sie trocken, getrübt, brüchig, käsig werden."

Ne umann (Lehrb. der Hautkrankheiten, 1880, pag. 274) erklärt: „Auch die Nägel erkranken an Psoriasis - Psoriasis ungium - wobei anfangs nur weisse Punkte erscheinen, später werden die Nägel missfärbig, gelb, braun, verdickt, trocken, locker, leicht ablösbar, zerklüften und brechen an ihrem freien Rande $a b$; unter dem Nagel bilden sich dichte Auflagerungen von Epidermis. Anderson, Csimmeskey beschreiben härtliche Massen, welche den normalen Nagel verdrängten."

Behrend (Lehrb. der Hautkrankheiten, 1883, pag. 262) äussert sich ähnlich dem Vorigen: „Nicht selten wird auch die Matrix der Nägel krankhaft verändert (Psoriasis ungium); es bilden sich in solchen Fällen unter dem Nagel anfangs kleine weisse Punkte, an denen es später zu dicken Epidermisbildungen und endlich zu Nutritionsstörungen des Nagelblattes kommt. Dasselbe wird glanzlos, missfarbig, dick, uneben, brüchig, aufgelockert und zerklüftet sich an seinem freien Rande."

H. von Hebra und Lesser erwähnen in ihren Büchern über Hautkrankheiten im Capitel Psoriasis der Nagelaffection 
gar nicht. Es erscheint das angemessen, wenn die Nagelsymptome nur secundäre, allen chronischen Hautkrankheiten mehr oder weniger zukommende wären.

Nach meiner eigenen Beobachtung muss man zwischen der primären Nagelerkrankung durch Psoriasis und der secundären Mitbetheiligung des Nagels bei Psoriasis streng unterscheiden; erstere ist als Psoriasis der Nagelmatrix (Psoriasis ungium im eigentlichen Sinne), letztere als eine Erkrankung der Nagelplatte in Folge einer im Nagelbette aufgetretenen Psoriasis anzusehen.

Die eigentliche primäre Psoriasis der Nägel ist ein Frühsymptom und findet sich sehr selten bei inveterirten Formen oder combinirt mit Psoriasis des Nagelbettes und deren Folgenzuständen auf den Nagel. Wo letztere Affectionen auftreten, ist meist die Nagelplatte so verändert, dass von jener primären Psoriasis ungium kaum etwas gesehen werden kann. Namentlich bei jugendlichen Individuen ist die primäre Erkrankung des Nagels relativ häufig anzutreffen. Der Sitz dieser Erkrankung ist ausschliesslich die Nagelmatrix, das hintere centrale Drittel desjenigen Bezirkes, der als Lunula durch seine helle Farbe sich abgrenzt (H. Hebra). Nach Zurückschieben des Nagelfalzes sieht man in diesem hinteren Drittel der Lunula eine Anzahl lebhaft roth gefärbter Pünktchen, welche auf Druck rorübergehend erblassen (siehe Figur I p). Einmal sah ich auch in der vorderen Hälfte der Lunula zwei derartige rothe Punkte. Obwohl im vorderen Theile der Lunula keine Papillen vorkommen, hat es den Anschein, als ob jene rothe Punkte hyperämischen Papillenköpfchen entsprächen, die wir nach jenem einen Befunde in der vorderen Hälfte der Lunula als neu gebildet uns zu denken hätten, was beim psoriatischen Processe durchaus nicht unwahrscheinlich gelten kann. Wir sehen wenigstens in Schnitten von psoriatischer Haut nicht nur Vergrösserung vorhandener Papillen, sondern auch wohl ausgebildete Papillen an Stellen, an welchen normaliter keine und fast keine Papillen auftreten, ferner am Grunde grosser verbreiteter Papillen neue papillenmässige Buchtungen verschiedener Grösse sowie Papillen, welche dichotomisch sich verzweigen und wieder neue Papillen erzeugen, ähnliche Vor- 
gänge wie beim Papillom und Hautcarcinom. Die rothen Punkte im Bereiche der Lunula halte ich bei Psoriasis für etwas Charakteristisches, soviel mir bekannt, keiner anderen Dermatose Zukommendes. Da diese pathologische Veränderung der Nagelmatrix angehört, so muss sie auch auf die Nagelplatte einwirken. Diese Einwirkung kennzeichnet sich im Verlaufe der Erkrankung als Tüpfelung der Nagelplatte (siehe Figur I $t$ ). Wo bei Psoriasis Tüpfelung der Nagelplatte gefunden wird, muss jene beschriebene Erkrankung der Lunula vorausgegangen sein, und oft genug wird im Laufe einer längeren Beobachtung in der Lunula, wenn sie durch wiederholtes Zurückschieben des Nagelfalzes frei gehalten wird, besagtes Anfangssymptom sich zeigen.

Wie die Tüpfelung der Nagelplatte einen Folgezustand der Nagelmatrixerkrankung darstellt, so erscheinen die secundären Frkrankungen des Nagels (Trübungen, Längs- und Querleisten, Verdickungen, Brüchigkeit), wie sie bisher als Nagelsymptome bei schwerer Psoriasis beschrieben sind, als Folgezustände einer psoriatischen Erkrankung des Nagelbettes. Diese secundären Nagelaffectionen nehmen meist ihren Anfang an der unter den beiden seitlichen Ecken des freien Nagelrandes befindlichen Haut, und erst später treten neue Herde centralwärts im Nagelbette auf (siehe Figur I s). Es tritt an den Nagelecken eine gelbliche hornartige, später käsige, bröckelige Verdickung der Haut und der Nagelplatte ein, welche sich nach rückwärts und nach der Mittellinie des Nazels zu nach und nach ausdehnt. Seltener sah ich, wie F. Hebra angibt, zu Beginn central im Nagelbett Psoriasisefflorescenzen als dunkle Flecken durch die Nagelplatte durchschimmern. Die weiteren gröberen nun folgenden Veränderungen für den Nagel sind so häufig baschrieben, dass ich nicht darauf zurückzukommen brauche. Nur möchte ich noch auf meine Beobachtung hinweisen, dass auch letztere von ihrem jeweiligen Ausgangspunkt gleichsinnig mit dem Vorwärtswachsen des Nagels sich verschieben. Hieraus folgt für die Onychopathologie, dass, wenn auch die Bildung der Nagelplatte der sog. Nagelmatrix ausschliesslich zukommt (Reichert, Ammon, Unna, H. Hebra), doch die Unterhaltung der Nagelplatte rom 
Nagel b e t t mitabhängt. Abgesehen von der Psoriasiserkrankung wird dies auch durch die analoge Veränderung der Nagelplatte bei Eczemen und Dermatomykosen dargethan, bei welchen die Nagelmatrix öfter als bei Psoriasis gesund bleibt. Tch glaube daher nicht, dass die mit dem fortwachsenden Nagel sich verschiebenden Veränderungen der Nagelplatte von einer Alteration der Nagelmatrix ausschliesslich herrühren müssen, oder umgekehrt bei Nagelbetterkrankungen (ohne Betheiligung der Nagelmatrix) die Nagelplatte intact bleibe, sondern vielmehr, dass jene secundären Nagelerkrankungen, trotz ihrer gleichsinnigen Verschiebung mit dem vorwärts wachsenden Nagel, ebensowohl von einer im peripheren (distalen) Theil des Nagelbettes erzeugten Störung verursachtsein können. Gerade Unnas Erwägungen ${ }^{1}$ ) bieten hinreichend plausibele Erklärung hierfür.

Eine Abhebung, Druckwirkıng, Austrocknung, Zerkliuftung des Nagelbettes muss bei der innigen Verbindung, welche die Nagelplatte mit dem Nagelbett während des Lebens hat, Folgen für den Nagel selbst hinterlassen. Bei jeder Operation eines Unguis incarnatus mit Entfernung der Nagelplatte sehen wir, dass der Nagel mit dem Nagelbette organisch zusammenhängt. Ausserdem wissen wir es aus anatomischen Untersuchungen, ${ }^{2}$ ) so dass die Nagelplatte nicht als eine blosse schützende Ueberdachung des Nagelbettes gelten kann.

In einem Falle universeller Psoriasis, bei welchem ich noch 2 Recidive bis zum Schwund der Symptome behandelte,

1) Unna. Anatomisch-physiologische Vorstudien zu einer künftigen Onychopathologie. Vierteljahresschr. f. Derm. u. Syph. 1881, p. 5, 12 u. f.

Siehe auch: Unna, Beiträge zur Histologie und Entwicklungsgeschichte der menschlichen Oberhaut und ihrer Anhangsgebilde. Arch. f. mikr. Anatomie. Bd. XII. 1876. Ref. Viertelj. f. Derm. u. S. 1876, p. 411.

Ferner: Unna. Beiträge zur Onychopathologie. Viertelj. f. Derm. u. Syph. 1877, p. 3.

H. Hebra. Beitrag zur Anatomie des Nagels. Wiener med. Jahrb. 1880. 1. u. 2. Heft. Ref. Viertelj. f. Derm. u. Syph. 1880, p. 309.

H. Hebra. Ueber Hyperkeratosis subungualis. Monatsh. f, prakt. Derm. Bd. VI. 1887. Nr. 7. (Eine Beschreibung, welche in den meisten Symptomen der secund. Nagelerkrankung bei Psoriasis genau entspricht.)

$\left.{ }^{2}\right)$ ef. Heynold. Beitrag zur Histologie und Genese des Nagels. Virch. Arch. LXV. 1875. Ref. Vierteljahrschr. f. Derm. u. S. 1876, p. 405. 
entstand beim letzten Ausbruch der Hautkrankheit ein subacuter Gelenkrheumatismus der grossen Gelenke. Wie bei früheren Attaquen waren auch hierbei sämmtliche Finger- und Zehennägel secundär erkrankt, und bei dem letzten complicirten Auftreten der Psoriasis fielen sämmtliche Nägel aus. Es fand aber nach und nach Wiederersatz statt. Ein Armgelenk heilte mit theilweiser Ankylose (Hebungsfähigkeit bis zur Horizontalen). Aehnliche Fälle sind in grosser Anzahl kürzlich noch von Bourdillon beschrieben. ${ }^{1}$ )

Die Mittheilung dieses eigenthümlichen Auftretens der Psoriasis möge Anlass sein, auf einige andere Begleitsymptome zurückzukommen, die ich bei frischen Ausbrïchen beobachtete: Ich konnte mich in einem Falle von dem jedesmaligen Auftreten einer Bronchitis gleichzeitig mit dem Auftreten zweier Recidive bei derselben Person überzeugen. Ein anderes Mal traf ich ischialgische Schmerzen. Sollte nicht der Psoriasisprocess doch auf Schleim- und serösen Häuten Veränderungen erzeugen, die dort als Catarrh oder einfache Hyperämie verlaufen, weil zu Verhornungs- und Abschuppungsvorgängen nicht Gelegenheit gegeben ist, und so die Ueberzeugung unserer französischen Collegen von einer arthritischen u. s. w. Diathese rechtfertigen? Warum sollen nur die Papillen der Haut erkranken bei einem multiplen und so ausgebreiteten Process? Fänden da nicht auch die von Hebra u. v. A. gemeldeten neuralgischen, rheumatischen etc. Symptome eine Erklärung? Hoffentlich gibt hier einmal eine mikroskopische Untersuchung neues Licht!

Nur zweimal fand ich Gelegenheit, Psoriasisstellen am Lebenden auszuschneiden und zur Untersuchung zu verwenden: einmal eine grosse alte, aber hochrothe schuppige Stelle aus dem Rücken links unter der Scapula, sodann eine ebenfalls schon ältere Stelle vom Oberarm. Beide Präparate gehörten jungen Männern an. Die betreffenden Patienten hatten, wenn auch kurze Zeit, der Behandlung mit Arsen und Chrysarobin unterstanden, was ich mit Rücksicht auf die Untersuchungen von

1) Bourdillon (Psoriasis et arthropaties, Thèse, Paris 1888). 
Robinson ${ }^{\mathbf{1}}$ ) und Jamies on ${ }^{\mathbf{2}}$ ) zu erwähnen nicht unterlassen will. Jedesmal excidirte ich weit im Gesunden, um Controlobjecte in den Randpartien direct zu erhalten. Die Härtung geschah zunächst nach Fle m ming dann in Alkohol steigender Concentration. Dem absoluten Alkohol pflege ich stets grössere Mengen frisch geglühten, gepulverten Kupfervitriols zuzusetzen, wodurch man sehr rasch zuverlässige Härtungsresultate bekommt. (Beiläufig erwähnt sei, dass ein solcher Alkohol ganz allein gebraucht, ausgezeichnet die Kerntheilungen fixirt, wenn man nicht zu grosse Stücke einträgt). Die Einbettung geschah in Celloidin. Die Mikrotomschnitte wurden $10 \mu$ dick.

Schon bei makroskopischer Betrachtung eines grossen Uebersichtsschnittes in einer schwarzen Schale roll Wasser kann man eine Anzahl Veränderungen wahrnehmen: zu oberst eine nach dem Centrum des Psoriasisherdes sich schräg verbreitender, weisser, homogener Streifen. Mit der Lupe sieht man, dass er dem Rete und seinen Zapfen entspricht und etwa auf das Fünffache seiner ursprünglichen Dicke in Maximo sich ausdehnt; sodann darunter eine äusserst helle, zarte durchsichtige Schicht, etwa dreimal so breit als die vorige, in gleichem Sinne mit der vorigen in schräger Richtung an Mächtigkeit wachsend; endlich hierunter eine weissliche, sehnig-opalisirende Schicht des derben Bindegewebes mit mancherlei Lï̈cken und gröberen Faserzügen.

Die Schnitte wurden zur Feststellung der allgemeinen histologischen Details z. Th. in Essigkarmin gefärbt und mit schwefelsaurem Eisen geschwärzt nach $\mathrm{O}$. Z a charias ${ }^{3}$ ), eine Methode, die ich ${ }^{4}$ ) anderen Orts bereits ausfiuhrlich beschrieb und empfahl. Ausserdem wurden angewandt: Carbolfuchsin und Carbolmethylenblau, Differenzirung durch mit $\mathrm{SO}_{4} \mathrm{H}_{\mathbf{\alpha}}$ angesäuertem Alkohol, Gabett's Lösungen I. und II. in verschiedener Dauer,

I) A. R. Robinson. Arch. of Dermat. Bd. VI. 1873, ef. Ziemssen. Handb. d. Hautkrankh. I. 1883, p. 502.

2) Jami es o n. Fdinb. med. Journ. 1879, p. 62 of. Z i e m s s e n ebenda.

3) O. Zacharias. Verhandl. d. Bremer Naturforscher-Versammlung 1891 , p. 121.

4) J. Schütz. Kurze Mitheilungen über bequeme Tinctionen fixirter Präparate. Monatsh. f. prakt. Dermatologie 1892. Bd. XIV. p. 397. 
Anilinwasser-Gentiana und Entfärbung nach Gram, Anilinwasser-Saffranin differenzirt in salzsaurem Alkohol, Hämatoxylin nach Ehrlich und Benda, Pikrokarmin und HCl-Alkohol. Dabei wurden öfters die Befunde an ungefärbten Präparaten in Glycerin, mit und ohne vorherige Behandlung mit verdïnnter Kalilauge, verdünnter Essigsäure, Aether-Alkohol controlirt.

Uebereinstimmend in beiden Fällen ergab sich nun Folgendes:

Die superficielle Hornschicht war, wie zu erwarten, sehr verbreitert. In ihrem obersten Theil ist sie in mehreren förmlichen Lamellen losgestossen (Fig. II und III). Weiter unten ist die Abhebung eine weniger zusammenhängende; dort sind die Zellen geringer in ihrem Zusammenhang gelockert. Dieser der Abschuppung bei Psoriasis entsprechende Befund erstreckt sich relativ weit nach unten in den centralen Partien des Präparats und zwar bis zur eigentlichen Stachelschicht. Soweit man an meinen Präpàraten daron reden kann, wird also auch die Körnerschicht, die Keratohyalin führende Zellengegend, mit in den Process der Abschuppung einbezogen. Ich sage, soweit man davon reden kann, denn in meinen Präparaten ist von einer scharf charakterisirten Körnerschicht nichts wahr. zunehmen. An einzelnen Stellen zeigen die Zellen gar kein Keratohyalin, an anderen Stellen ist das Vorkommen des Keratobyalins sogar ausgedehnter als normal. Immer handelt es sich aber um nur spärliche Einlagerungen von Keratohyalin, nie fand ich Zellen, die auch nur annähernd einem normalen Verhalten entsprachen, und als Schichte ist eine Keratohyalin führende Zone überhaupt nicht zu entdecken. Die Beziehungen des Keratohyalins zu den sog. Spiralen der Epithelien konnte ich vorläufig wegen der Fixirung meiner Präparate in Flemming'scher Lösung nicht verfolgen, da die Weigert'sche Fibrinfärbemethode für derartige Präparate nicht zı verwenden ist. Ein gesetzmässiges Verhalten des Keratohyalins gegenüber der Färbbarkeit der höher gelegenen Zellkerne oder unter den betreffenden Zellen etwa versammelter Wanderzellen, wie es Kromayer ${ }^{1}$ ) angibt, konnte ich an meinen Schnitten nicht

1) Kromayer. Zur pathol. Anatomie der Psoriasis, Arch. f. Derm. u. Syph. 1890 , p. 557 a. f. 
herausfinden. Dass der Abschuppungsprocess so tief herunterreicht, sieht man aus der Gleichartigkeit des zelligen Verhaltens und der Gleichheit der verschiedenen Tinctionen in fraglichen Schichten.

Es handelt sich um verdickte Zellmäntel, deren Inneres kein Protoplasma, keinen Kern mehr birgt. Nur in den alleruntersten Reihen, die der einstmaligen Körnerschicht entstammen, findet sich manchmal am Rande einer centralen Vakuole ein wandständiger, halbmondförmiger Kernrest. Grade die Färbung nach $\mathrm{Z}$ acharias, die sich für alte, verhornte Partien trefflich eignet, zeigt diese Kernreste noch deutlich.

Somit fängt die eigentliche Haut, die mit dem Körper noch in organischem Zusammenhange steht, nach unseren Präparaten bei Pscriasis erst mit der Stachelschicht an.

Diese Stachelschicht ist über den Papillengipfeln nicht besonders mächtig, oft sogar von geringer Höhe, bis auf drei übereinanderliegende Zellenlagen verschmächtigt. Dieser verschmächtigte Theil der Stachelschicht über den Papillenspitzen führt an vielen Stellen in Haufen zusammenliegende Wanderzellen mit sich, die sich allerdings auch in den Lamellen der sich loslösenden Hornschicht, wie schon $\mathrm{Kromayer}^{1}$ ) an Schnitten von Psoriasisschuppen sah, gruppenweise und alternirend finden. $\mathrm{Ob}$ in der That ein Zusammenhang zwischen Abhebung von Hornzellen und der in der obersten Stachelzellenschicht sich ansammelnden Leukocyten existire, konnte ich nicht nachweisen. Vereinzelte Leukocyten werden, wie bei vielen chronischen Hautkrankheiten, auch in der Tiefe, also überhaupt in dem Rete, gefunden. Auf ihren Wanderungen zwängen sie sich, so gut es angeht, zwischen den Interspinalräumen der Stachelzellen hindurch und nehmen dementsprechend die wunderlichsten, meist lang ausgezogene, S-förmige Figuren an, wie es schon von F. Pagenstecherr) abgebildet ist, und wie es analogerweise beim spitzen Condylom und

1) Kromayer. Zur pathol. Anatomie der Psoriasis. Arch. f. Derm u. Syph. 1890, p. 557 u. f.

$\left.{ }^{2}\right)$ F. Pagenstecher. Ueber die Eatwicklung der Epithelzellen bei chron. Hautkrankheiten und dem Epithelialcarcinom. Aus d. LVII. Bd der Sitzungsber. d. Wiener Akad. II. Abth. Aprilh. 1868. Sep.-Abz. 
Epithelialcarcinom - bier regelmässig in unübertroffener Anzahl - zu sehen ist. ${ }^{1}$ )

Diese vereinzelten. Leukocyten zeigen namentlich in den tieferen Lagen der Schleimschicht auch Kerntheilungen („Fragmentirung(") in all den Formen, wie sie der verstorbene Arnold ${ }^{2}$ ) auf reichen Tafeln zusammengestellt hat.

Die Retezapfen sind im Centrum des Krankheitsherdes enorm verlängert und verbreitert, theilweise zu mehreren verschmolzen. Einige zeigen an ihrer unteren Spitze neue Buchtungen, obwohl eine ausgesprochene Tendenz zu dichotomischen Theilungen, wie beim Papillom, Carcinom, beim Lupus verrucosus der Extremitäten es Sitte ist, nicht zu bemerken ist. Die Stachelzellen sind bis zu den basalen Cylinderzellen herab von der Erkrankung mit ergriffen. In jeder Höhe des Rete treffen wir mitunter Zellen, deren Kerne von einer lichten Höhle umgeben sind. (Fig. IV.) Die interspinalen Räume sind stellenweise stark erweitert, so dass die Stachelsäume schon bei 100facher Vergrösserung sich scharf abheben. Für meine Präparate muss ich daher ein Oedem des Rete annehmen. Die basalen Cylinderzellen erscheinen vermehrt und je nachdem man frischere oder ältere Stellen besichtigt, auf drei und mehr übereinanderliegende Lagen angewachsen. Thre Form ist die einer lang ausgezogenen Spindel, deren grösste Breite den Kern umschliesst (Fig. IV). Auch unter ihnen gibt es solche, welche bereits Vacuolenbildung um den Kern herum zeigen (Fig. IV). An Carbolfuchsinpräparaten sind diese Verhältnisse besonders schön zu sehen. An der Tinction nehmen diese Cylinderzellen sehr ungleichen Antheil; es gibt solche, die sehr dunkel gefärbt erscheinen. Letztere bilden durch mehrere Schichten ein fortlaufendes Ganze (Fig. IV) und stehen offenbar in besonderem Zusammenhang.

1) J. Schütz. Mikroskopische Carcinombefunde nebst ätiologischen und praktisch verwendbaren diagnostischen Ausblicken. (Mit 6 Mikrophotographien.) Frankfurt 1890.

2) Arnold. Weitere Beobachtungen über die Theilungsvorgänge an den Knochenmarkzellen und weissen Blutkörperchen. Virch. Arch. Taf. IV. Bd. XCVII. Sep.-Abz.

Arnold. Ueber Kern- und Zelltheilung bei acuter Hyperplasie der Lymphdrüsen und Milz. Virch. Arch. Bd. XLV. Taf. II, Fig. 9 etc. S.-A. cf. auch Photogramm $\nabla$. meiner citirten Krebsarbeit. 
Mitosenbildung ist im Rete reichlich anzutreffen und besonders schön in den Essigkarmin-Eisen-Präparaten hervortretend. Aber nie sah ich Mitosengruppen, nie Grössenunterschiede, pathologische Formen. Sie werden an der ganzen Peripherie der Retezapfen in den untersten Zellenlagen, vorzüglich oberhalb der Cylinderzellen angetroffen. Eine besondere $\mathrm{Be}-$ vorzugung seitens der karyokinetischen Zellen geniessen die unteren Spitzen der Retezapfen und die den Papillenspitzen correspondirenden Thäler des Rete (cf. Fig. III m). Analoges habe ich auch beim Papillom und Carcinom wahrgenommen, was uns ein Längenwachsthum der Epithelzapfen in diametraler Richtung erklärt. Da bei Psoriasis eine ständige Abgabe von Zellen durch den Schuppungsprocess unterhalten wird, ist diese Wahrnehmung ron Wichtigkeit.

Der Papillarkörper und seine Papillen sind bedeutend verbreitert und verlängert. Gegen den von einzelnen Autoren allen Ernstes gegen eine solche Behauptung gemachten Einwurf der Möglichkeit einer durch schräge Schnittführung erlebten Täuschung brauche ich mich im Hinblick auf meine Figuren II und III, sowie meine makroskopischen Wahrnehmungen wohl nicht zu salviren. Oft ist der Gipfel der Papille kolbig erweitert, das basale Ende durch Aneinanderrücken der Retezapfen stielartig eingeengt. Angelegte Serien lassen auch hier Täuschungen ausschliesen. Auffallender Weise fand ich in den Papillen sehr wenig erweiterte Capillarschlingen voll rother Blutkörperchen, wie ich a priori an meinen in Flemming'schen. Gemisch gewesenen Hautstäckchen bei einer Psoriasisstelle erwartet hatte, und wie ich sie sonst (bei fixirten Präparaten von zahlreichen Carcinomen, Papillomen, Lupus erythematosus, Warzen) stets fand. Nichts hiervon! Den kolbigen Hohlraum der Papille nimmt oft nur ein enorm erweitertes Lymphgefäss ein, das einzelne polynukleäre Leukocyten beherbergt. Oder man sieht an Stelle desselben bei schärfster Einstellung ${ }^{1}$ ) ein

1) Namentlich bei Trockensystemen muss der justirten Tubuslänge und der Deckglascorrectur entsprochen worden sein, sonst erscheint die Zeichnung zu blass für eine ausreichende Beobachtung. Es ist ev. nöthig, an solchen Stellen die Deckglasdicke nach $\mathrm{Zeiss}$ (Catalog 1889, p. 26) zu bestimmen. Ich habe mir eine Zahl 0,015 ein für allemal gemerkt, mit 
feines Maschenwerk (bei Präparaten, welche in Carbolfuchsin tingirt und mit Glück in $25 \% S O 4 H^{2}$-Alkohol kurz differenzirt wurden). An der Spitze des Lymphgefässes gewahrt man nicht selten eine grössere kreisrunde Oeffnung. Begleitet ist dieses Lymphgefäss ron wanderlustigen emigrirten Rundzellen, geschwänzten Bindegewebszellen und blassen ovalen, feingranulirten kleinen Plasmazellen. Eine sogenaunte kleinzellige perivasculäre Infiltration mit scharfen, runden Kernen und wandständigen dunkeltingirten Nucleolis sah ich nicht.

Die kollagene Substanz im subpapillären Theil, welcher den horizontalen Gefässzügen zum Bette dient, erscheint gequollen, die Bindegewebsbündel auseinandergedrängt. Controlpräparate normaler und pathologischer Haut, die ich aus meiner Sammlung: $z u$ Rathe $z o g$, sowie extra angefertigte Präparate vorhandenen schnittfertigen Materials verschiedener Provenienz, welche ich gleichen Bedingungen aussetzte, liessen mir hieriber keinen Zweifel aufkommen. Bei schwacher Vergrösserung sieht man allerdings auch in der subpapillaren Schicht oft mächtige Zellenziige (Fig. V), welche grösseren, meist horizontal laufenden Gefässen folgen. Auch diese erwiesen sich zum Theil nicht als gefüllte Blutgefässe, sondern Lymphgefässe mit einer Masse fixen geschwänzten Bindegewebszellen, zarten Plasmazellen wenig ausgewanderten Rundzellen (Fig. V b, p, l) um sie herum. Keineswegs handelt es sich auch hier um Zellen resp. Kerne, wie sie die sogenannte regelrechte kleinzellige Infiltration der Hauptsache nach darstellen (cf. Fig. V r). Der geringe. Bestand an gefüllten erweiterten Blutgefässen ist mir auch in diesen tieferen Schichten sehr aufgefallen.

Trotz allen Suchens konnte ich ausser der Fragmentirung bei den Wanderzellen an den im Bindegewebe zerstreuten Zellen Mitosen nirgends auffinden. (Einmal fand ich im centralen Theil einer Talgdrüse, mitten in der Stützsubstanz eine Metakinese.) Allerdings weiss ich aus Erfahrung, dass es bei Lupus und sonstigen Infiltraten hiermit überhaupt seine Schwierigkeiten hat. Mastzellen traf ich höchst selten an. Den

der ich die Theilstriche der Mikrometerschraube, welche beim Einstellen aufs Präparat und Deckglasoberseite durchlaufen werden, multiplicire, um annähernd richtigen Stand für. die Correctionsfassung sofort zu wissen. 
Quersebnitten von Nerven und Gefässen, denen ich begegnete, konnte man durchaus nichts Pathologisches nachsagen.

Eine Ueberraschung wurde mir indess bei Darstellung der elastischen Fasern zu theil, als ich deren feinen Ausläufern in den Papillen nachging. Hier sah ich eine so reichliche und zarte Fadenbildung, die sich schnurstracks zu den Epithelzellen hinwandte, dass es bei mir feststand, diese Dinge womöglich elektiv zur Cognition zu bringen. Ich musste mir sagen, dass die bisherigen Methoden hierzu zu zeitraubend für mich waren und anch ein jedesmaliges Probiren benöthigten. So verliess ich die Färbungen nach Tänzer, Unna, Mibelli und Martinotti und entschloss mich, auf eigener Bahn mich dem Glück anzurertrauen und hierbei namentlich den feinsten Ausläufern des elastischen Organs nachzuspüren. Nach mannigfachen Fehlgriffen und Versuchen erhielt ich befriedigende Aufschlïsse nach folgenden Verfahren:

1. Härtung im Flemming'schen Gemisch - Nachhärtung in Alkobol - (Härtung in Alkohol allein ist für die später zu beschreibende Färbung unvortheilhaft) - Celloidinschnitte von $10 \mu$ Dicke - Aufbewahrung derselben in $90 \%$ Alkohol.

2. Zur elektiven Färbung der elastischen Fasern werden 2 Raumtheile kalt gesättigter, wässriger Pikrinsäurelösung mit 1 Raumtheil Carbolfuchsinlösung in ein Reagensglas gebracht, geschïttelt und in ein Schälchen gegossen. Das entstandene Gemisch ist schmutzig dunkelgrün. S o fort nach der Mischung wird der zu färbende Schnitt in die Farbflotte mittels Spatels aus dem Spiritus übertragen. Bei leisem Aufsetzen des Spatels auf die Oberfläche der Farblösung schwimmt der Schnitt ausgebreitet auf derselben. Die Farblösung darf dann nicht mehr erschüttert werden. Es ist dies wichtig, weil später die Farbflüssigkeit einen klebrigen Bodensatz abscheidet, mit dem der Schnitt nicht in Berïhrung kommen darf. Der Schnitt verweilt circa 5 Minuten in der Farbe. Alsdann wird er mit dem Spatel herausgehoben und in eine grosse Krystallisationsschale voll Wasser übertragen, indem man ebenfalls den Spatel lose auf die Oberfläche des Wassers aufsetzt, worauf der Schnitt sofort ausgebreitet bleibend davonschwimmt. Sogleich nimmt der Schnitt eine rosenrothe Farbe an; ebenso färbt sich das 
Wasser. Alsbald wird der Schnitt auf dieselbe Weise in eine zweite Schale mit neuem Wasser befördert und bleibt darin einige Minuten, um Pikrinsäure abzugeben und sich intensiv zu färben. Alsdann hebt man den Schnitt mit dem Spatel aus dem Wasser, lässt das Wasser vom Spatel abtropfen, saugt mit einer Ecke Fliesspapier noch etwas Wasser ab und entwässert ganz kurz in absolutem Alkohol. Je rascher letzteres gelingt, um so besser für die Tinction, da der Alkohol sehr viel Farbe entzieht. Rasch bringt man den Schnitt in reichlich Cedernöl, lüftet denselben mit einer Glasnadel von seiner Unterlage etwas und sorgt dafür, dass er bald auf der Oberfläche des Oels sich glatt ausbreite. Die hierbei sich losstossenden Tropfen rothgefärbten Alkohols schiebt man mit einem Glasstab an die Wandung des Schälchens und wartet, bis der Schnitt untersinkt. Alsdann schiebt man ein Deckglas unter den Schnitt, hebt ihn auf diesem heraus, drückt wiederholt eine Schicht Fliesspapier auf ihm ab und bettet schliesslich auf erwärmtem Objectträger in purem Canadabalsam ein.

Entzieht der Alkohol zu viel Farbe, so kann man auch den Schnitt aus dem Wasser auf das Deckglas bringen, mit Fliesspapier trocknen und Deckglas mit Schnitt für 6 bis 8 Stunden in Petroleum legen und dann in Cedernöl und Canadabalsam bringen. Die Kohlenwasserstoffe des Petroleums entfernen den Wasserrest, ohne Farbe zu extrahiren. Die Entwässerung in Petroleum hat sodann auch den Vorzug, dass hierbei keine Schrumpfungserscheinungen wie bei der Wasserentziehung durch Anilinöl und Anilin-Xylol auftreten, ein Factor, der grade bei elastischen Fasern zu beachten ist, da sonst hier farbenprächtige Kunstproducte hervorkommen. Für jeden Schnitt muss man eine nicht zu geringe Menge frischen Petroleums anwenden.

Auf riel einfachere Weise, wenn auch nicht ganz so sicher kann man die elastischen Fasern nach Härtung in Flemmingsgemisch an Psoriasisschnitten nachweisen, wenu man 5 Minuten in Carbolfuchsin (Gabett's Lösung I) färbt, in Wasser abspült, 2 bis 3 Secunden in saurer Methylenblaulösung (Gabett's Lösung II) resp. in $25 \% \quad \mathrm{SO}_{4} \mathrm{H}_{2}-$ Wasser entfärbt, wieder in Wasser abspïlt und so rasch wie möglich in Alkohol trocknet. 
Letzteres Verfahren hat sogar den Vorzug einer etwas distincteren Kern- und Zellfärbung.

Mit Hilfe dieser Färbungen nun gelang es mir stets, das elastische Organ: in einer Weise bei den Psoriasispräparaten zur Anschauung zu bringen, wie ich es nie gesehen hatte.

Von dem im subpapillaren Theil der Cutis meist horizontal und schräg ziehenden dicken elastischen Fasern, welche sich an die kollagenen Bündel anschmiegen oder von den die Haut durchsetzenden glatten Muskeln ihren Ursprung nehmen, zweigen sich feinste Fasern ab, welche zu allem, was den Namen Zelle führt, ziehen, um direct mit derselben zu verschmelzen. Die Richtungen der Fasern wechseln mannigfach. Zahlreiche Verzweigungen, anastomosenartige Verbindungen geben dem ganzen Fasernetz ein eigenartiges Aussehen. An den Zweigstellen sieht man oft, wie ein Raupennest zwischen einer Zweiggabelung ausgespannt, die Sederholm'scheMémbranen (Fig.V m). ${ }^{1}$ ) So bekommen die geschwänzten Bindegewebszellen Fasern zugetheilt,- welche direct mit den Ausläufern jener Zellen sich verbinden. So ergeht es den Talg- und Schweissdrüsenzellen, den Haarbulbis, so den Zellen, welche die Gefässe bilden, so den Bindegewebszellen, welche diese begleiten.

Am Fusse der Papillarschicht umziehen die elastischen Fasern rings die Retezapfen (Fig VI und VII), dann aber steigen aus kurzen Bögen feinste, gerade gespannte Reiser auf, welche zum Theil in der Papille mit den Gefässen senkrecht emporziehen, zu den Epithelien aber kaum erkennbare zahllose Fädchen abgeben.

Diese Endfasern senken sich direct in das Epithel ein. Hier scheinen sie (mit Immersion verfolgt) 2 bis 3 und mehr Zellenlagen weit die schon erwähnte ungleiche Tinction der Zellmäntel zu bewirken und namentlich die Zellgrenzen mit einem tieferen Colorit (durch Addition ihres eigenen) zu beglücken, dann aber sich in die feine grade Streifung des Stachelmantels aufzulösen (Fig. IV). Wir lernen hier gelegentlich der Psoriasisuntersuchungen das elastische Organ der Haut

1) Sederholm. Einige Untersuchungen über die Anordnung des elastischen Gewebes in der Haut. Ref. in Monatsh. f. prakt. Derm. 1891. II. p. 520 . 
als ein Zwischenstuick zwischen Epithel und Bindegewebe, überhaupt als eine ständige Verbindung aller Zellen kennen, das durch seinen Wechsel in der Spannung die Ernährung, Formation, gegenseitige Lage der Zellen beeinflussen muss. Hat man erst einmal an wohlausgebildeten Psoriasisplaques diese Darstellurig des elastischen Organs erreicht, so findet man auch an normaler Haut und anderen Präparaten diese Verhäitnisse in Kleinen wieder, stets dieselben Analogien, aber nicht so grossartig wie hier. Ungezwungen kommt man zu der Erkenntniss, dass das elastische Organ der Haut bei Psoriasis wie die Zellen selbst zu einer vermehrten Entwicklung gelangt ist.

Besonders beachtenswerth erscheinen breite Retezapfen, an deren unterer Basis, resp. verbreiterten Spitze neue Abtheilungen, secundäre Retezapfen zu bilden sich beginnen. Ganz junge Stadien dieser Art (Fig. VII) zeigen die entstehende secundäre Papille noch ganz leer von Blutgefässen und Bindegewebszellen, aber schon mit einem respectablen Convolut charakteristisch sich anordnender feinster elastischer Fasern ausgerüstet, die von den resp. zu den Epithelien ziehen, ein bedeutsames Factum fuir die Papillengenese. Ein wichtiges Zeichen für die Betheiligung des elastischen Organs am Psoriasisprocess.

Die Prognose und Therapie der Psoriasis gab wenig Anregung unter meinen Fällen Neues zu sammeln.

Prognostisch erschienen stets Fälle am günstigsten, welche noch nicht behandelt waren, oder nur wenige Recidive durchgemacht hatten.

Häufige Recidive, öfteres Erkranken derselben Körperstelle, unvollkommen durchgeführte Curen pflegten die Abheilung der Erkrankung hinauszuschieben. Complicirende Eczeme in Folge zu starker Einwirkung der äusseren Therapie oder bei individueller Unzuträglichkeit derselben gab durch die Verzögerung der Heilwirkung die Maxime, bei jedem Patienten und neuen Psoriasisausbruch anfangs die Mittel in niederen Procenten anzuordnen und den Grundsatz zu verfolgen: „Lieber viel zu wenig als etwas zu viel!" Der Rückschlag, welcher bei letzterer Eventualität eintritt, die künstlich entstehenden neuen Schübe von Psoriasis, welche durch die Reizung desselben Wittels erzeugt werden, das zu ihrer Zerstörung dienen soll, 
brauchen eine Zeit, dass, wenn sie nur ganz selten sich ereignen, jede Statistik über die Zeitdauer über den Haufen geworfen wird. Jedenfalls steht das Unheil, das durch unvorsichtige Dosirung angerichtet werden kann, in gar keinem Verhältniss zu dem durchschnittlichen Zeitverlust, den ein moderirtes Vorgehen bei Durchführung der Curen mit sich bringt. Eine leere Statistik kann hier wenig nützen; Erfahrung und Schaden für Patienten und Arzt geben die Lehre, einen langsamen Verlauf ohne Zwischenfälle überraschenden Erfolgen vorzuziehen. Die Localisation der Efflorescenzen hat bei frischen aber in der Ausbreitung stillstehenden Krankheitsfällen, wie ich mich sieben Mal überzeugen konnte, einen unverkennbaren Einfluss auf die Rückbildung, insofern die periphersten Ausläufer der Psoriasis viel später zur Involution kommen als die central gelegenen, wenn nicht besondere Schädlichkeiten (Druckeinwirkung von Kleidern, Verbänden, Bruchbändern u. a. m.) eine Gegenwirkung unterhaiten.

Unter der, wie wir sahen, berechtigten Ausscheidung exceptioneller Fälle beanspruchte die Therapie eine Zeit von 4 bis 11 Wochen. Die Behandlung selbst verblieb, ausser den vorbereitenden Einwirkungen von Wasser, Seife, Fett und luftabschliessenden Bedeckungen überwiegend den alterprobten Mitteln: Arsen, Theer, Chrysarobin und Pyrogallussäure, welche bis auf ganz verschwindende Ausnahmen nie versagten bei consequentem Gebrauch und richtiger Auswahl. Für Arsen war der primäre Ausbruch von Psoriasis und die grosse Dosis (ev. ohne äusserliche Behandlung) wiederholt empfehlenswerth erschienen. Die neueren und neuesten Mittel: Jodkalium, Hydroxylamin, Aristol etc. zeigten sich für die Privatpraxis so wenig angebracht, dass ich gerne nach wenigen Anwendungen zum Früheren zurückkehrte, nicht ohne mich von einer factischen Einwirkung dieser Substanzen auf die Heilung der Psoriasis überzeugt zu haben und mancherlei Vorzüge der Präparate anerkennen zu müssen. Für den Mangel an praktischem Werth. welchen die vielen neuen Arzneikörper gegenüber den verbreiteten älteren genannten Mitteln in der Privatpraxis haben, musste uns vorläufig die grosse theoretische Bedeutung entschädigen, dass die Rubricirung unserer Psoriasismittel überhaupt unter den einheitlichen Begrift des Reductionsmittels 
durch den neuen Zuwachs gegen Psoriasis wirkender Substanzen gefestigt wurde. Mit doppeltem Interesse liest man (Binz, Vorlesungen über Pharmakologie) jetzt die Formeln, nach welchen Jodkalium seine Sauerstoffentziehung geltend macht, Arsen und Phosphor reducirend wirken, Pyrogallussäure seine vielbeklagten Färbungen durch energische Sauerstoffoccupation bewirkt, Chrysarobin bei Anwesenheit von Alkalien zu Chrysophansäure oxydirt. Eines Mittels, das meines Wissens recht selten angewendet wird, muss ich bier noch mit einer besonderen Dankbarkeit gedenken, weil es in 2 Fällen die bestehende individuelle $\mathrm{Un}$ verträglichkeit für die meisten reducirenden Mittel nicht theilte,

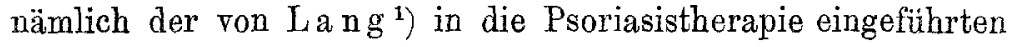
Rufigallussäure. Obwohl die Behandlung eine recht lange Dauer in Anspruch nahm, konnte sie doch bis zum Schluss obne neu hinzutretende Störungen durchgeführt werden. In einem ähnlichen neuen Falle würde ich an erster Stelle auf diese Substanz zurückgreifen. Complicirende Arznei-Conjunctivitiden erlebte ich nur zweimal. (Ich lasse Nachts Handschuhe tragen, Schutzbrillen werden meist auf die Dauer refusirt.) Die Augencatarrhe schwanden in einigen Tagen unter schwachen Zinkkokaineinträufelungen $(0,075: 0,15: 20,0)$ und halber Dunkeleur. Gegen complicirende Chrysarobineczeme leisteten Un nas Einleimungen ${ }^{2}$ ) gute Dienste.

Endlich habe ich über zwei Patienten zu berichten, deren spontane Befreiung der Psoriasis des ganzen Körpers, die ja häufiger gelegentlich ron Kranken behauptet wird, jedesmal auf Ferienreisen im Höhenklima eintrat. Beide Patienten gehörten dem Gelehrtenfache an, so dass an leichtfertige Behauptungen hierbei nicht gedacht werden kann.

Wenn wir uns zum Schlusse aus dem Rahmen des nackten Thatsächlichen und der trockenen Statistik herausbegeben und zurückblickend auf das Gesammelte zu einjgen Erwägungen und Schlussfolgerungen anschicken, so sei ron vorneherein bemerkt, dass es durchans mir fern liegt, in den Fehler zu

1) E. Lang. Ueber Psoriasis, Schuppenflechte. Volkmanns Sammlung kl. Vortr. 1881, Nr. 208, p. 1785.

¿) Unna. Aerztl. Vereinsbl. f. Deutschland. 1886, p. 427. 
fallen, eigene Befunde mit gegentheiligen anderer bei dieser Erkrankung in irgend welche Opposition za stellen und mich an der negativen Kritik zu betheiligen, die seit Anbeginn in der Geschichte der Psoriasis - nicht gerade zum Vortheile hervortritt. Eine Erkrankung, welche so vielgestaltig auftritt wie die Psoriasis, bald nur wenige punktförmige Herde, bald grosse Flächen des Körpers besetzt; bald langsam und rein local, dann stürmisch und unter Umständen mit Begleiterscheinungen auftritt, die bei dem einen núr eine leicht gelbe Farbe der Efflorescenzen zeitigt, bei den meisten starke Röthung und Neigung zu Blutung bewirkt, die central in einer Efflorescens von selbst abblassen und heilen kann, 'die geringe und wieder überaus mächtige Schuppenlager erzeugt, die überdies in hohem Masse vom jeweiligen Allgemeinzustande des Patienten beeinfiusșt werden kann und geographisch sogar Symptomenunterschiede zeigt, bei dieser können unmöglich alle Befunde der Autoren übereinstimmen. Namentlich finden wir dies für die Histologie bestätigt; denn, wie verschieden sind hier die Angaben! Wo widersprechen sich mehr mikroskopische Bilder, wenigstens scheinbar?' Die gänzliche Unkenntniss von der Aetiologie und der Wesenheit der Psoriasis muss uns mahnen, bei dieser Vielgestaltigkeit uns vorläufig eher auf das Sammeln von Positivem und Einzelbetrachtungen $\mathrm{zu}$ verlegen, als die Aufstellung weitreichender Theorien anzustreben, deren Consequenzen stets ein Theil sich nicht gutwillig einfügender Darstellungen zum Opfer fällt, um ganz oder theilweise in Vergessenheit zu gerathen.

Naturgemäss werden unsere Betrachtungen zunächst den beiden Cardinalsymptomen geltén: der Schuppung und Röthung.

Die. Psoriasisschuppen haben klinisch und differentialdiagnostisch das Kennzeichen, dass sie nie kleienartige Abscbilferungen bilden, sondern dass die. Psoriasisschuppe stets eine zusammenhängende Lamelle kleineren oder grösseren Umfangs darstellt, nach deren Abhebung eine glänzende rothe, nur durch das bekannte Bulkley'sche Psoriasishäutchen gedeckte Stelle erscheint, welche leicht zu verletzen ist und dann blutet. Im Einklange hiermit stehen unsere Befunde an der Hornschicht. Wir sahen, dass die Ausbreitung der lamellösen 
Abhebung sehr tief, bis zum Anfangen der Stachelzellen reichte und sich horizontal über grössere Strecken ausbreitete, wobei immer grosse Zellenmassen in Zusammenhang blieben. Erklärt ist auch nach den mikroskopischen Befunden die leichte Verletzlichkeit und das leichte Eintreten der Blutungen; denn wir fanden über den hohen Papillengipfeln manchmal nur wenige Lagen mehr oder weniger weicher protoplasmatischer Stachelzellen und eventuell dariuber ein nur geringe Mengen Keratohyalin führendes stratum granulosum, ebenso häufig auch gänzliches Fehlen des letzteren. Da die Verhornung in dem kaum als stratum aufzufassenden Bereich der Körnerzellen eine höchst unvollkommene ist, erscheint die leichte Verletzbarkeit wohl begründet.

Nicht so leicht ist die bald starke, bald weniger ausgesprochene Röthe der Psoriasis nach unseren Schnitten zu erklären, und es entsteht die Frage, ist die Röthe lediglich auf Kosten der von vielen Autoren gemeldeten Erweiterung papillärer Blutgefässe und deren strotzenden Füllungszustand zu setzen? An unseren eigenen Präparaten fanden wir für eine Bejahung dieser Frage keinen Anhalt, sondern die a priori erwarteten Zeichen der Hyperämie waren keineswegs deutlich. Hier bleiben nur 2 Befunde zur Beantwortung:

1. Die schon genannte Verdünnung der Haut über dem gefässtragenden Theile der Haut und 2. die Verminderung des Keratohyalins bezï̈glich der ausgebliebenen Verhornung.

Wir wissen, dass die Körner des stratum granulosum es sind, welche die weisse Farbe der menschlichen Haut erzeugen, indem sie wie die Fettkügelchen in der Milch das Licht reflectiren (Unna).

Wo das Keratohyalin mehr oder weniger fehlt, wie am Nagelbette, dem Lippenrothe, ${ }^{1}$ ) den Schlëmhäuten, sehen wir die Haut lebhaft roth, auch wenn kein pathologisch starker Füllungszustand der Gefässe vorhanden ist. Umgekehrt sehen wir bei den sonderbarerweise "Psoriasis buccalis" genannten

1) Lažansky (zur Keratohyalinfrage, Verhandlgen. d. Deutschen dermatolog. Ges. 1889, pag. 236) fand im Epithel des Lippenrothes im Gegensatz zu Unna's Behauptung Körnerzellen. 
weissen Stellen der Wangenschleimhaut, wie Schuchardt') es abgebildet hat, pathologisch starke Keratohyalinbildung eintreten. Diese Factoren genügen, um zu zeigen, dass, wenn von mir u. A. Präparate gesehen wurden, in denen keine strotzenden Füllungszustände der Blutgefässe hervortraten, doch eine klinische Röthe vorhanden sein kann.

Es ist somit wahrscheinlich, dass ausser dem Füllungszustande der Gefässe bei Psoriasis die rothe Färbung der Haut mit durch die fehlende Horndecke und den mangelhaften Gehalt der Körnerzellen an Keratohyalin bedingt ist.

Vielleicht ist eine geringe Betheiligung der Körnerschicht an dex nur gelblichen Färbung der Psoriasisstelleu Schuld, welche man bei schlecht ausgebildeter Psoriasis, wie bei dem erwähnten Fettleibigen, in seltenen Fällen zu Gesicht bekommt.

Das im Rete vorhandene Oedem (Kernhöhlen in den tiefer gelegenen Stachelzellen, erweiterte Interspinalräume), die Quellung der collagenen Substanz, die erweiterten Maschen des tieferen Bindegewebes, die Erweiterung der Lymphgefässe liefern eine plausibele Entstehungsursache für die leichte Convexität, welche frische Psoriasisplaques oft zeigen.

Gleichzeitig sind dieselben wohl der Grund für eine durch. zu reichliche Ernährung unterhaltene Zellwucherung im Rete und Bindegewebe, welch' erstere durch zahlreiche Mitosenbildung direct $\mathrm{zu}$ verfolgen ist.

Beziehungen der Leukocyten zur Zellwucherung waren bei den Psoriasispräparaten nicht zu beweisen, ebenso wenig wie zum Schuppungsprocesse.

Woher die erweiterten Lymphgefässe, woher das Oedem, dem der Charakter eines entzündlichen Oedems nicht beigemessen werden kann, da die Begleiterscheinungen von Entzündung acuter oder chronischer Art (kleinzellige Infiltration, reichliche Mastzellen, Narbenbildung) fehlen? Erhöhter Druck, vermehrte Blutzufuhr, verminderte Abfuhr, erleichterte Transsudation durch Verdünnung der Blutmasse können hier in Betracht kommen.

1) Schuchardt. Beiträge zur Entstehung der Carcinome aus chronisch entzündlichen Zuständen der Schleimbäute und Hautdecken. Habilitationsschrift. Leipzig 1885, p. 16. 
1. Eine Verdünnung der Blutmasse kann man wohl schon deshalb ausschliessen, weil dort, wo eine solche vorkommt und solange sie vorkommt, Psoriasis schwindet, andererseits Psoriasis gerade robuste Naturen heimsucht.

2. Staung activer Art ist schwer anzunehmen, da gerade in der Haut reichliche Collateralen sich finden ${ }^{\mathbf{1}}$ ) und die Venen stets viel weiter sind als die zugehörigen Arterien. Eine a cu te active Hyperämie würde sich daher nicht leicht einstellen. Psoriasis tritt aber manchmal sehr acut auf, folglich kämen wir auf

3. die Ursache zurück, welche überhaupt in $7 / 8$ aller Fälle Grund der Oedeme ist: passive Stauung, und es gälte näher zu erfahren, ob Venen oder Lymphgefässe oder beide betheiligt sind, wie es bei den meisten Oedemen der Fall ist.

Aus den Präparaten scheint hier nur soviel zu eruiren zu sein, dass jedenfalls die Lymphgefässe mit einen Antheil haben. Bei der Annahme der passiven Staung würde manches verständlich sein, z. B.: die Abbeilung der Psoriasis bei Wasserbehandlung und Oeleinreibungen nach $\mathrm{Lang},{ }^{2}$ ) welche die Haut erschlafft, in gleicher Weise die Heilungen im Bade Leuk, die günstige Wirkung des Höhenklimas, durch die absaugende Wirkung der forcirt athmenden Lungen.

Die vorwiegende Localisation der Psoriasis an Stellen, welche eine straffe Anheftung an eine Unterlage haben oder die, wie an den fixen Punkten der Articulationen, im Wachsthume gegenüber der Umgebung zurückbleiben, finden vielleicht eine Erklärung in den an diesen Stellen vorhandenen complicirten Gefässschlingen. An all' diesen Stellen gibt es Talgdrüsen und meist zugehörige Haare; wo diese aber sind, sind auch complicirte Gefässe. Sodann häufen sich dort elastische Fasern an, die, wie wir sahen, auch nach unseren Präparaten überreich vorhanden sind und $\mathrm{zu}$ den durch Psoriasis veränderten Zellen in directer Beziehung stehen. Freilich kann erst eine allgemeiner geübte regelmässige Untersuchung des elastischen Organes an normaler und pathologischer Haut

1) Spalteholz. Ueber die Blutgefässe der Haut. Ergänzungsheft z. Archiv f. Derm. u. Syph. I., 1892, pag. 112.

$\left.{ }^{2}\right)$ Lang l. c. und Vierteljahrschr. f. Derm. u. Syph. 1880, p. 475. 
definitiven Aufschluss geben, wie weit das pathologische Verhalten der elastischen Fasern reicht.

Die oft zu beobachtende Symmetrie der Psoriasisefflorescenzen, welche den mannigfachsten Theorien über Psoriasis eine willkommene Stütze war, mag immerhin auch bei unseren Betrachtungen als Beweismittel gelten, dass der Psoriasis irgend welche anatomisch begründete prädisponirende Momente als Unterlage dienen. Es können unsere anatomischen Annahmen mit dem gleichen Rechte wie sonstige frühere durch das symmetrische Verhalten gestützt werden.

Hand- und Fusssteller; wo das Vorkommen echter Psoriasis zu den Ausnahmen gehört, sind nach $U n n a, N$ e umann ${ }^{\mathbf{1}}$ ) am ärmsten an elastischen Fasern. Schleimhäute, an denen Psoriasis mindestens selten Erscheinungen veranlasst, haben ungleich weniger elastische Fasern als die äussere Haut (Frey). ${ }^{2}$ )

An Hand- und Fussteller sind sodann nach Tom s a ${ }^{3}$ ) sehr wenig Hautmuskeln vorhanden, die auf das elastische Organ und Gefässe einwirken können, wohl aber ausserordentlich weite Venen, welche Staungen nicht förderlich sind ${ }^{4}$ ), die meisten Lymphgefässe (Neumann), ${ }^{5}$ ) viele Collateralen (Spalteholz). ${ }^{6}$ )

Von den Streckseiten wissen wir, dass sis gegenüber den Beugeseiten in der Haut weniger Papillen enthalten (Werthheim), ${ }^{7}$ ) also auch weniger papilläre Gefässe.

Das erste Auftreten der Psoriasis zur Zeit der Veränderung in der Hautspannung beim Wachsen des Organismus spricht. ebenfalls für die Annahme, dass die Psoriasis an den mehrfach genannten fixen Oertlichkeiten der Haut passiven Gefässstaungen ihr Dasein verdanken kann.

Die offenkundige häufige Heredität der Psoriasis könnte leicht einer Prädisposition zur Last fallen, welche durch eine vererbte anormale Ausbildung des elastischen Organs entstiunde.

1).Unna. Ziemssens Handb. d. Hautkrankh. I. p. 15; Neumann, Lehrb. d. Hautkrankh. 1883, p. 2.

$\left.{ }^{2}\right)$ Frey. Histologie und Histochemie. 1876, p. 246.

3) Tomsa. Vierteljahrschr. f. Derm. u. Syph. V. p. 16.

4) Ebenda.

5) N eumann. Vierteljahresschr. f. Derm. u. Syph. V. p. 147.

6) Spalteholz l. c.

${ }^{7}$ W Wrtheim. Vierteljahresschr. f. Derm. u. Syph. VIII. p. 334. 
Hierfür spräche u. a. auch das häufigere Befallensein von Leuten "mit straffen Muskelfasern" (Hebra), das seltenere Vorkommen beim weiblichen Geschlecht, kleinen Kindern, Schwächlingen, der oft männliche Typus von Weibern, die an Psoriasis leiden.

Auch die Complication der Psoriasis mit Akne, Furunkeln, Lichen pilaris, die Seltenheit genuiner Eczeme bei Psoriasispatienten verweist auf eine derbe Veranlagung der Haut, wie sie als "Aknehaut" gegenüber der zarten "Eczemhaut" den Dermatologen bekannt ist.

Die nahen Beziehungen der elastischen Fasern zum Wachsthum des Epithels sahen wir bei den beschriebenen Secundärpapillen anatomisch ausgedrückt.

Da das elastische Organ an den Hautmuskeln seinen Anfang nimmt (Unna), ${ }^{1}$ ) und die weitere Annahme Unnas von seinem Zweck, zu grosser Anfüllung der Lymphräume mit Gewebsflüssigkeit automatisch zu steuern, plausibel erscheint, so würden die Reizresultate K öbner's und Wurtz d offer's ohne Schwierigkeiten mit dem bisher Entwickelten in Einklang zu bringen sein.

Unser Fall, der mit Gelenkrheumatismus gleichzeitig an Psoriasis erkrankte und zwar so heftig, dass sämmtliche Zehenund Fingernägel in kurzer Zeit ausfielen, spricht sehr dafür, dass von Psoriasis in seltenen Fällen auch andere Häute als die Epidermis (seröse Häute, Schleimhäute) in ihrer Weise mitbefallen werden können.

Die Häufigkeit, mit der manche ausländische Autoren solche Gelenkaffectionen, ferner rheumatische Beschwerden, Combinationen von nerrösen Symptomen, Catarrhe und asthmatische Beschwerden sahen, legen ein Weitergehen des Processes auf genannte Membranen sehr nahe. Dass hierbei andere Symptome, wie auf der Haut eintreten können, ist aus der verschiedenartigen Structur genannter Membranen klar. Eine analoge ödematöse Durchtränkung und Verdickung der Membranen würde aber vollständig die Symptome und bei Gelenken auch eine Veranlassung zu Entzündung erklären.

1) Unna 1. c. p. 14. 
Es läge nahe, noch weiter durch verbindende Gedanken das Vorhandene zu ergänzen. Ich muss hiervon absehen, um nicht zu sehr in rejp hypothetisches Gebiet zu gelangen, während es meine Absicht war, nur die vorhandenen Befunde zu geben und lediglich an diese anlehnend Einzelerwägungen anzuschliessen, die unbekümmert um subjective Anschauungen von dem Wesen der uns beschäftigenden Krankheit verwerthbar sein möchten.

Sollte dieser Zweck wenn auch nur in dem einen oder anderen Punkte für eine dereinst entstehende gefestigte Theorie von der Aetiologie äer Psoriasis erreicht werden, so würde ich die meinen Aufzeichnungen zu Grunde liegende Mühe reich belohnt sehen.

\section{Erklärung der Abbildungen auf Taf. XIII.}

Fig. 1. An Psoriasis erkrankter Fingernagel eines kleinen Mädchens (Lupenvergrösserung). $\quad p=$ primäre Erkrankung der Nagelmatrix. $t=$ Prim. Erkrank. der Nagelplatte ("Tüpfelung"). $\beta=$ Beginnende secundäre Nagelerkrankung bei Psoriasis: Verdickung und Gelbfärbung des Nagels an den Ecken, Streifungen der Nagelplatte.

Fig. 2. Schnitt aus der Randzone einer Psoriasisefflorescenz des Rückens. - Essigkarmin-Eisen-Präparat. - Geringe Abhebung der Epidermis. - Beginnende Papillenvergrösserung. - Zellenzüge in der Cutis. - Zeiss, Apochromat. 8 Mm. Comp. Ocular 4, Abbe's Camera.

Fig. 3. Stelle aus der Mitte desselben Präparates. - Starke lamellöse Abhebung der Epidermis bis zur Stachelschicht, die hier auch über den Papillen noch sehr mächtig ist. - Mittelstarke Papillen- und Retezapfenvergrösserung. - Mitosen im Rete. - Lymphgefässe begleitende Zellzüge. - Dieselbe Vergrösserung und Färbung wie bei Fig. 2.

Fig. 4. Basale Cylinderzellenschicht seitlich an einem Retezapfen. - Mehrere Lagen derselben. - Vacuolen um den Kern und im Protoplasma. - Verbreiterte Interspinalräume. - Elastische Fasern dringen von der Papille aus ins Epithel, sich in die Zellgrenzen und den ge. streiften Stachelmantel der Zellen verlierend. - Carbolfuchsin-Methylenblau-Präparat. - Zeiss, Immersion-Apochromat. $2 \mathrm{Mm}$. Comp. Oc. 4.

Fjg. 5. $b=$ geschwänzte Bindegewebszellen. $p=$ Plasmazellen. $l=$ Leucocyten mit, z. Th, angedeuteten Fragmentirungen. $r=$ Rundzellenkerne aus eirrem Lupusinfiltrat. $m=$ Gabelung einer elastischen Faser mit Sederholm'scher Membran. - Zeiss, Apochr. 4 Mm. Comp. Oc. 4.

Fig. 6. Elastische Fasern in der Cutis und einer Papille. $m=\mathrm{Mi}-$ tose, $b$ = fixe Bindegewebszellen; Carbol-Euchsinpräparat. - Zeiss, Apochromat 4 Mm., Comp. Oc. 4.

Fig. 7. Unteres Ende eines grossen Retezapfen mit secundärer Papillenbildung. Letztere enthält nur elastische Fasern, keine Gefässe. Picrinsäurefuchsin-Präparat. Vergrösserung wie bei Fig. 6. 
言
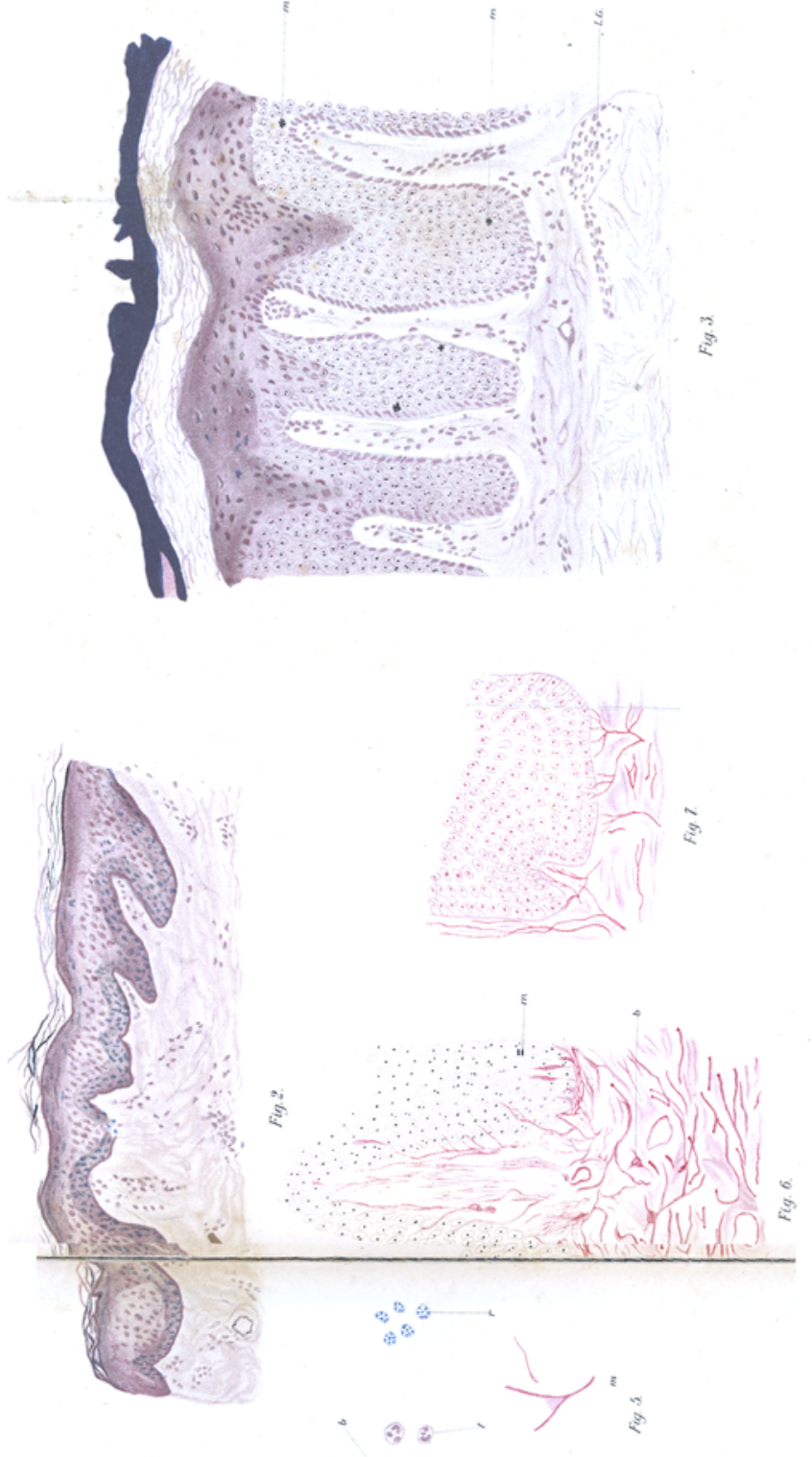

3

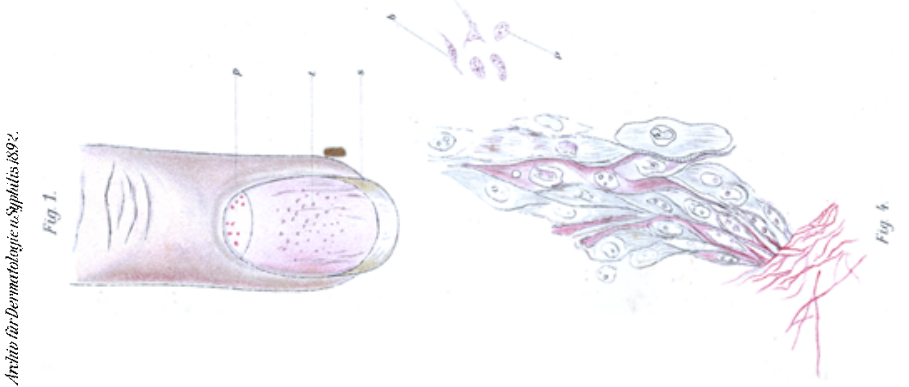

